\title{
Bildung für nachhaltige Entwicklung und inklusive Bildung - Grundlagen, Konzepte und Potenziale
}

\begin{abstract}
Zusammenfassung
Das Sustainable Development Goal (SDG) 4 „Hochwertige Bildung - Inklusive, gerechte und hochwertige Bildung gewährleisten und Möglichkeiten des lebenslangen Lernens für alle fördern" bezieht sich gleichermaßen auf Bildung für nachhaltige Entwicklung (BNE) und inklusive Bildung. Während BNE vor allem darauf zielt, Individuen zum Umgang mit einer nachhaltigen Entwicklung zu befähigen, steht bei inklusiver Bildung die Möglichkeit zu Teilhabe und Partizipation an Bildung und Gesellschaft für alle Menschen im Fokus. Bisher sind die wissenschaftlichen Diskurse zu BNE und inklusiver Bildung allerdings eher getrennt voneinander geführt worden. Die Entwicklung einer gemeinsamen Perspektive auf BNE und inklusive Bildung stellt aktuell ein Desiderat dar. Vor diesem Hintergrund bezieht dieser Beitrag die Konzepte einer BNE und inklusiver Bildung aufeinander und betrachtet, welche Potenziale sich für eine gemeinsame Fundierung, aber auch die praktische pädagogische Umsetzung hieraus ergeben.
\end{abstract}

Schlüsselworte: Bildung für nach haltige Entwicklung, Inklusive Bildung, Inklusive BNE, Vielfalt, Umgang mit Komplexität

\footnotetext{
Abstract

The Sustainable Development Goal (SDG) 4 "Quality Education - Ensure inclusive and equitable quality education and promote lifelong learning opportunities for all" refers equally to Education for Sustainable Development (ESD) and Inclusive Education. While ESD aims primarily to empower individuals to deal with sustainable development, inclusive education focuses on the opportunity for all people to participate in education and society. To date, however, scientific discourses on ESD and inclusive education have tended to be conducted separately. The development of a common perspective on ESD and inclusive education is still needed. Against this background, this paper relates the concepts of ESD and inclusive education to each other and discusses the resulting potentials for a common foundation, but also the practical pedagogical implementation.
}

Keywords: Education for sustainable development, inclusive education, inclusive ESD, diversity, coping with complexity

\section{Einleitung}

Bezieht man das Sustainable Development Goal (SDG) 4 „Hochwertige Bildung - Inklusive, gerechte und hochwertige Bildung gewährleisten und Möglichkeiten des lebenslangen Lernens für alle fördern“ und die „Convention On The Rights Of Persons With Disabilities“ (United Nations, 2006) aufeinander, so wird deutlich, wie relevant und aktuell Bildung für nachhaltige Entwicklung (BNE) und inklusive Bildung und deren gemeinsame Umsetzung sind. Inklusion fordert einen gleichberechtigten Zugang zu Bildung für alle und das Erkennen sowie Überwinden von Barrieren; BNE möchte Nachhaltigkeitskompetenzen aller Lernenden entwickeln.

Bisher sind die wissenschaftlichen Diskurse zu BNE und inklusiver Bildung allerdings eher getrennt voneinander geführt worden, obwohl es sich bei beiden um Konzepte handelt, die für das gesamte Bildungssystem von hoher Relevanz sind. Die Entwicklung einer gemeinsamen Perspektive auf BNE und inklusive Bildung stellt noch ein Desiderat dar (Böhme, 2019). Es gibt bisher nur wenige Publikationen zum Verhältnis von BNE und inklusiver Bildung und auch Materialien für die praktische Bildungsarbeit sind nur wenige entwickelt worden. Eine Ausnahme stellen hier die Unterrichtsreihen dar, die von Behinderung und Entwicklungszusammenarbeit e.V. (bezev) entwickelt worden sind (Svinos, 2019; Westermeier, 2015). ${ }^{1}$

Vor dem Hintergrund des SDG 4 stellt sich insofern im Sinne von hochwertiger und effektiver Bildung die Aufgabe, BNE und inklusive Bildung aufeinander zu beziehen, Zusammenhänge herzustellen, Vorreiterprojekte zu präsentieren und die beiden Konzepte gemeinsam in den Strukturen des Bildungssystems und der Bildungspraxis zu verankern (KMK \& BMZ, 2015, S. 56f.). Hierfür sind die Forschung und Entwicklung in Wissenschaft und Praxis voranzubringen. Theorien, Konzepte und Materialien können entwickelt werden; hier 
besteht noch viel Potenzial. Dies könnte auch die Pädagoginnen und Pädagogen entlasten, die z.B. in der Schule BNE und inklusive Bildung bisher als zwei nebeneinanderstehende Querschnittsthemen und somit als zusätzliche Herausforderungen wahrnehmen. Hier stellt sich unter anderem die Frage, wie Lehrkräfte so ausgebildet werden können, dass sie sowohl in der Lage sind, inklusive Klassen zu unterrichten, als auch die Entwicklung von Nachhaltigkeitskompetenzen bei allen Schüler/-innen unter Berücksichtigung der Heterogenität in diesen Klassen zu fördern. Ziel ist es, Bildung für nachhaltige Entwicklung allen zugänglich zu machen und im Sinne des Whole School Approach Bildungseinrichtungen in allen Bereichen und auf allen Ebenen gemäß BNE und inklusiver Bildung zu gestalten.

Mit Blick auf eine gemeinsame Perspektive auf BNE und inklusive Bildung lässt sich die folgende Fragestellung aufwerfen, die für die Reflexion in diesem Beitrag leitend ist: Wie können die beiden Konzepte zum gegenseitigen Mehrwert aufeinander bezogen werden? Damit einher gehen folgende Teilfragen:

- Gibt es gemeinsame normative Fundierungen sowie Ziele einer BNE und inklusiver Bildung?

- Wie kann BNE in der methodisch-didaktischen Umsetzung inklusiv gestaltet werden?

- Inwiefern gibt inklusive Bildung Anregungen für die Möglichkeiten und Grenzen der individuellen Entwicklung von hochkomplexen Nachhaltigkeitskompetenzen?

- Welche Kompetenzen benötigen Pädagoginnen und Pädagogen für BNE und inklusive Bildung?

Bevor eine Auseinandersetzung mit diesen Fragen stattfindet, wird das jeweilige Verständnis von BNE und inklusiver Bildung dargelegt.

\section{Bildung für nachhaltige Entwicklung}

Seit Ende der 1990er Jahre wird in der bildungswissenschaftlichen Diskussion sowie der Bildungspraxis verstärkt Bezug auf eine nachhaltige Entwicklung genommen. In diesem Kontext ist das Konzept einer Bildung für nachhaltige Entwicklung (BNE) entwickelt worden (Rieckmann, 2016; Michelsen \& Fischer, 2015).

Ohne Lernprozesse wird eine nachhaltige Entwicklung nicht möglich sein (Vare \& Scott, 2007). Daher soll Bildung für nachhaltige Entwicklung dazu beitragen, eine nachhaltige Entwicklung der Gesellschaft zu fördern. Sie zielt darauf $a b$, Menschen zu befähigen, sich an den gesellschaftlichen Lernund Verständigungsprozessen für eine nachhaltige Entwicklung, der Umsetzung der SDGs und damit der Förderung einer „Großen Transformation“ (WBGU, 2011) zu beteiligen.

Lernende sollen - als „Nachhaltigkeitsbürger*innen“ (Rieckmann \& Schank, 2016; Wals \& Lenglet, 2016) - in die Lage versetzt werden, ,eine nachhaltige Entwicklung mitzugestalten und ihre eigenen Handlungen diesbezüglich kritisch zu reflektieren“" (Künzli David, 2007, S. 35). Dazu gehört v.a. auch die Fähigkeit zur kritischen Auseinandersetzung mit einer nachhaltigen Entwicklung und mit der Komplexität, der Unsicherheit sowie den Widersprüchen, die mit ihr verbunden sind.
Dieser emanzipatorische Ansatz einer Bildung für nachhaltige Entwicklung sieht als das wesentliche Bildungsziel die Entwicklung von Schlüsselkompetenzen an, die Individuen zu einer aktiven Gestaltung des Prozesses einer nachhaltigen Entwicklung befähigen. In diesem Zusammenhang werden die folgenden Nachhaltigkeitskompetenzen als besonders relevant erachtet: Kompetenz zum Vernetzten Denken, Kompetenz zum Vorausschauenden Denken, Normative Kompetenz, Strategische Kompetenz, Kooperationskompetenz, Kompetenz zum Kritischen Denken, Selbstkompetenz, Integrierte Problemlösekompetenz (Rieckmann, 2018; UNESCO, 2017). Zudem wird von einer Bildung für nachhaltige Entwicklung ein Beitrag zu einem kritischen Wertediskurs erwartet (Rieckmann et al., 2014; Rieckmann \& Schank, 2016). Sie kann und soll Anregungen geben, die eigenen Werte zu reflektieren und Stellung zu nehmen in der Wertedebatte auf dem Weg zu einer nachhaltigen Entwicklung. Ihr Potenzial liegt somit auch darin, den Wertehorizont der Lernenden zu erweitern. Bildung für nachhaltige Entwicklung geht davon aus, dass Lernprozesse selbst als Partizipationsprozesse zu gestalten sind und sich an Partizipation zu orientieren haben (Rieckmann \& Stoltenberg, 2011). Denn Kompetenzen können nicht einfach gelehrt oder unterrichtet werden; sie müssen selbst entwickelt werden (Weinert, 2001). Bildung für nachhaltige Entwicklung bedarf daher einer handlungsorientierten, transformativen Pädagogik (Rieckmann, 2018; UNESCO, 2017), die sich durch didaktische Prinzipien wie z.B. Lernendenzentrierung und Zugänglichkeit, Handlungs- und Reflexionsorientierung, Partizipationsorientierung, Vernetzendes Lernen, Visionsorientierung und transformatives Lernen auszeichnet (Künzli David, 2007; Littledyke \& Manolas, 2011; Rieckmann, 2018; UNESCO, 2017).

Diese Innovationen im Lehren und Lernen setzen allerdings bei den Lehrenden neue Lehr-Lernkompetenzen voraus (Bertschy, Künzli \& Lehmann, 2013; Vare et al., 2019). Die Lehrenden müssen idealerweise selbst Nachhaltigkeitskompetenzen besitzen und diese bei ihren Lernenden entwickeln können. Das bedeutet, dass sie ein kritisches Verständnis von nachhaltiger Entwicklung auf der einen Seite und des pädagogischen Ansatzes von Bildung für nachhaltige Entwicklung auf der anderen Seite haben müssen. Sie benötigen Wissen über innovative Lehr-Lernmethoden, aber auch Fähigkeiten zu deren Anwendung. Sie brauchen zudem Fähigkeiten zur Begleitung der Lernenden, wozu auch gehört, die eigene Rolle als Lehrende kritisch zu reflektieren und sich selbst eher als Lernbegleiter/-in zu verstehen. Bildung für nachhaltige Entwicklung geht es nicht nur darum, eine nachhaltige Entwicklung in die Lehre zu integrieren und z.B. Schulfächern oder Studiengängen neue Inhalte hinzuzufügen. Schulen und Hochschulen sowie auch andere Bildungseinrichtungen sollen sich als Orte des Lernens und der Erfahrung für eine nachhaltige Entwicklung verstehen und daher alle ihre Prozesse an Prinzipien der Nachhaltigkeit ausrichten. Damit BNE wirksamer ist, muss die Bildungseinrichtung als Ganzes verändert werden. Ein solches ganzheitliches Konzept (Whole Institution Approach) zielt darauf ab, Nachhaltigkeit in alle Aspekte der Bildungseinrichtung (Curriculum, Betrieb, Organisationskultur, etc.) zu integrieren. Auf diese Weise fungiert die Institution selbst als Vorbild für die Lernenden (UNESCO, 2017). 


\section{Inklusive Bildung}

Seit der Ratifizierung der UN-Konvention über die Rechte von Menschen mit Behinderungen in Deutschland im Jahr 2009 ist Inklusion ein geltendes Recht. Die Europäische Union ist seit Beginn des Jahres 2011 daran gebunden und hat sich verpflichtet, die Umsetzung im Umfang ihrer Zuständigkeiten zu unterstützen. Die Konvention fordert in ihren 50 Artikeln umfassende Teilhabe von Menschen mit Behinderung und sieht inklusive Entwicklung als gesamtgesellschaftliche Aufgabe. Innerhalb dieses Beitrags ist besonders Artikel 24 in den Blick zu nehmen. Denn hier geht es um die Anerkennung des Rechts auf effektive Bildung für Menschen mit Behinderung. Damit wird eine lange Vorgeschichte an rechtlichen und normativen Entwicklungen verstärkt: Die Allgemeine Erklärung der Menschenrechte, der UN-Sozialpakt und die Kinderrechtskonvention sind Beispiele hierfür.

Während sich die UN-Konvention auf ein enges Verständnis von Inklusion bezieht und Menschen mit Beeinträchtigungen im Fokus sieht, orientieren sich viele weitere Dokumente und Regularien an einem weiten Inklusionsbegriff. Dabei geht es dann nicht nur um die Gruppe der Menschen mit Behinderung oder Beeinträchtigung, es werden zusätzlich viele weitere Diversitätsmerkmale wie z.B. Geschlecht, sozioökonomischer Status, Herkunft, familiärer Status oder Kultur berücksichtigt. Es wird von einer heterogenen Gesellschaft ausgegangen, die sich so entwickeln muss, dass jede Person abhängig von ihren Präferenzen, aber unabhängig von ihren Voraussetzungen an allen Bereichen des gesellschaftlichen Lebens partizipieren kann und dazu die notwendige Unterstützung erhält, bzw. die Konditionen so angepasst werden, dass der $\mathrm{Zu}$ gang ermöglicht wird.

Die rechtliche Lage ist also deutlich; die Umsetzung in der Realität bleibt in vielen Belangen jedoch weit hinter den Ansprüchen zurück. Vieles befindet sich auf dem Weg, und Inklusion ist als ein Prozess zu verstehen. Häufig wird die Diskussion zu inklusiver Bildung alleine auf den Kontext Schule bezogen. Der Wirkungsbereich inklusiver Bildung geht jedoch weit darüber hinaus und eine Veränderung der Schule alleine reicht nicht aus. Das macht folgende Definition des Projekts „BRIDGES - Werkstatt Inklusion“ deutlich: „INKLUSION bezeichnet Basiswerte der Gesellschaft: Partizipation ist ein Menschenrecht. Alle Menschen werden in ihrer Vielfalt und Individualität wahrgenommen, angenommen und wertgeschätzt. Die Vielfalt wird als Ressource wahrgenommen. Bezogen auf Bildung in der Schule und dort speziell im Unterricht bedeutet für uns Inklusion, auf Bedürfnisse von Gesellschaft und Individuen einzugehen sowie individuelle Lernvoraussetzungen zu erkennen, zu berücksichtigen und dementsprechend $\mathrm{zu}$ fördern. Individualisierung und Gemeinschaft sind dabei gleichermaßen wichtig" (Baumert, Vierbuchen \& Team BRIDGES, 2018, S. 526). Schule spielt im Bereich Bildung eine relevante Rolle; ihr kommt eine wegweisende Funktion in der Entwicklung einer inklusiven Gesellschaft zu. Hier kann der Whole Institution Approach aus dem Kontext BNE übertragen werden: Inklusive Bildung erfordert eine Veränderung der gesamten Schulstruktur und nicht nur die Ermöglichung eines Zugangs zur Schule für einzelne Schüler/-innen mit Behinderungen. In der Umsetzung von inklusiver Bildung spielen Lehrkräfte eine bedeutsame Rolle, denn wichtig ist, wie sie den
Unterricht und die Lernumgebung sowie das soziale Klima gestalten und die Voraussetzungen der Schüler/-innen einbeziehen. Doch was benötigen Lehrende für die Umsetzung inklusiver Bildung?

Die European Agency for Development in Special Needs Education (2011) stellt drei Dimensionen als bedeutsam für erfolgreiche Lehrkräftebildung heraus:

- Wissen (Knowledge):

Kennen effektiver Maßnahmen und Konzepte

- Handeln (Skills):

Erproben und Übernehmen der neuen Maßnahmen

- Einstellungen (Attitudes, Beliefs):

Reflexion sozialer Werte und Normen

Alle drei Dimensionen stehen wechselseitig in Interaktion (Gable, Tonelson, Park, Sheth \& Wilson, 2012) und sollten in Aus-, Fort- und Weiterbildung für Lehrkräfte berücksichtigt werden. Neben dem notwendigen Fachwissen müssen Lehrkräfte Wissen über die Entwicklung von Kindern und Jugendlichen und pädagogische Kompetenzen besitzen, um ihren Schüler/-innen passende Ansätze der Unterstützung und Lerngelegenheiten bieten zu können. Die Kenntnis solcher passenden und wirksamen Maßnahmen und Strategien spielt hier eine fundierende Rolle. Handeln kann in diesem Modell auch als Können verstanden werden: nicht nur Maßnahmen und Strategien kennen, sondern sie auch jeweils modifizieren und implementieren können. Und nicht zuletzt benötigen Lehrkräfte eine positive Einstellung gegenüber Inklusion und die Überzeugung, dass sie für alle Schüler/-innen verantwortlich sind. Die Arbeit an alleine einer dieser drei Komponenten ist nicht ausreichend; es benötigt diesen Dreiklang für erfolgreiche Lehrkräftebildung für Inklusion.

Im Kontext inklusiver Bildung müssen sich Lehrkräfte auch selbst als „lebenslang Lernende“ (European Agency for Development in Special Needs Education, 2011, S. 77) verstehen; sei es in der Nutzung und Anwendung von aktuellen Forschungsergebnissen oder in der Kooperation mit anderen Fachkräften. Kooperation und multiprofessionelle Teamarbeit gewinnen im Kontext inklusiver Bildung deutlich an Relevanz. Lehrkräfte und Schulen sind in ein breites Netzwerk mit internen und externen Akteur/-innen eingebettet. Lehrkräfte arbeiten mit anderen Lehrkräften oder pädagogischen Fachkräften zusammen, bereiten Unterricht gemeinsam vor, führen ihn durch und reflektieren ihn, um das Beispiel des Co-Teaching als einen möglichen Kooperationsbereich anzuführen.

\section{Inklusive Bildung für nachhaltige Entwicklung?}

Wie können die beiden umfassenden Konzepte einer Bildung für nachhaltige Entwicklung und inklusiver Bildung nun zusammengebracht werden? Die Notwendigkeit von Inklusion sowie einer nachhaltigen Entwicklung stellen Bildung vor neue Aufgaben: „Die Globalisierung benötigt genauso pädagogische Antworten wie der gesellschaftliche Wunsch, Menschen mit Behinderungen nicht weiter systematisch von gesellschaftlicher Teilhabe auszuschließen und der Vielfalt in der Gesellschaft diskriminierungsfrei zu begegnen“ (Führing \& Böhme, 2015, 
S. 2). Bisher finden Diskussionen und inhaltliche Auseinandersetzung zu beiden Herausforderungen allerdings meist noch parallel zueinander und ohne gegenseitige Bezugnahme statt. Mithin geht es darum, eine „Brücke zu bauen zwischen zwei pädagogischen Prinzipien, die geradezu prädestiniert sind, gemeinsam gedacht und umgesetzt zu werden “ (Führing \& Böhme, 2015, S. 2). Um auch Lernende mit Beeinträchtigungen zur Mitgestaltung einer nachhaltigen Entwicklung zu befähigen (Empowerment) und sie nicht von diesen Beteiligungsprozessen per se auszuschließen, bedarf es einer inklusiven BNE (Bhatia \& Singh, 2015; Svinos, 2019).

BNE und inklusive Bildung haben einen gemeinsamen normativen Kern: Für beide Konzepte ist der Gedanke des Empowerments zentral, und sie sind den Menschenrechten, der menschlichen Entwicklung und sozialer Gerechtigkeit verpflichtet (Böhme, 2019; Führing \& Böhme, 2015). Beiden Konzepten liegen demnach hohe ethische Ansprüche zugrunde, die es im Alltag zu leben gilt. In diesem Sinne kann BNE auch einen Beitrag zum Empowerment gegen Benachteiligung leisten: „Viele SchülerInnen mit sonderpädagogischem Förderbedarf oder Migrationshintergrund erfahren im alläglichen Leben strukturelle Gewalt in Form von Diskriminierung und Ausgrenzung. Für diese SchülerInnen ist es notwendig, Kompetenzen zu erlangen, die es ihnen ermöglichen diese strukturelle Gewalt als solche zu identifizieren und mögliche Handlungsoptionen kennen zu lernen" (Führing \& Böhme, 2015, S. 7 f.). Hier wird deutlich, dass es für eine nachhaltige Entwicklung und Inklusion einerseits um die Veränderung der Strukturen der Gesellschaft und des alltäglichen Lebens geht, aber andererseits eben auch darum, Kinder, Jugendliche und Erwachsene dabei zu unterstützen, Kompetenzen und Strategien für Partizipation und Mitgestaltung in einer komplexen Welt zu entwickeln.

Bezüglich der Ziele geht es der inklusiven Bildung um eine grundsätzliche gesellschaftliche Partizipation, während BNE speziell auf die Befähigung zur Mitgestaltung einer nachhaltigen Entwicklung abzielt. Somit kann inklusive Bildung als eine Voraussetzung für BNE betrachtet werden. Denn erst wenn eine Förderung aller Lernenden gemäß ihrer individuellen Bedarfe sichergestellt (und Zugänge zu Gesellschaft und Bildung für alle gewährleistet) ist, wird es möglich, dass alle entsprechend ihrer individuellen Voraussetzungen Nachhaltigkeitskompetenzen entwickeln und einen Beitrag zu einer nachhaltigen Entwicklung leisten können (Böhme, 2018).

Gemeinsamkeiten weisen die beiden Bildungskonzepte auch hinsichtlich einiger didaktischer Prinzipien auf. Denn sowohl BNE als auch inklusive Bildung weisen eine Lernerzentrierung, einen Lebensweltbezug und eine Handlungsorientierung auf und gehen von der Notwendigkeit der Differenzierung/ Individualisierung aus (Böhme, 2019; Westermeier, 2015). Während Führing und Böhme (2015) die Auffassung vertreten, dass Aktivitäten des Globalen Lernens „ohne größere Anstrengungen so gestaltet werden [können], dass SchülerInnen mit den unterschiedlichsten Lernvoraussetzungen einbezogen werden können" (S. 11), wird in diesem Beitrag die Meinung vertreten, dass diese Aufgabe der methodisch-didaktischen Umsetzung einer inklusiven BNE doch anspruchsvoller ist.

Zunächst einmal wird das Thema Classroom Management als übergreifendes Konzept auch für eine inklusive BNE an Bedeutung gewinnen. Wie kann der Klassenraum oder der
Lernort vorbereitet werden? Wie intensiv bereitet die Lehrkraft den Unterricht vor? Wie findet die Planung und Unterrichtung von Regeln und unterrichtlichen Verfahrensweisen statt? Wie kann ein positives (Lern-)Klima geschaffen und aufrechterhalten werden? Welche Verantwortlichkeiten werden welchen Schüler/-innen übertragen? Welche kooperativen Lernformen können eingesetzt werden? Vor allem proaktive Kriterien des Classroom Managements (eine Übersicht der Kriterien findet sich z.B. bei Hennemann \& Hillenbrand, 2010) sollten in einer inklusiven BNE mitgedacht werden. Zusätzlich sollten jedoch auch reaktive Kriterien, also Strategien für potenzielle Probleme, vorhanden sein.

Während in der BNE häufig durch sehr offene Unterrichtsmethoden die Thematik selbstentdeckend erarbeitet wird, wird hier bei Kindern mit kognitiven oder Verhaltensbeeinträchtigungen häufig eine Grenze erreicht. Für diese Zielgruppe muss BNE strukturierter und ggf. auch in einem anderen Lerntempo bearbeitet werden. Der Lernprozess sollte „zeitlich und personell so flexibel gestaltet werden, dass ein individueller Lernerfolg aller SchülerInnen sichergestellt werden kann" (Böhme, 2019, S. 241). Deutlich wird im Folgenden, dass inklusive BNE viele Chancen und Möglichkeiten bietet, jedoch, falls diese nicht genutzt werden (können), auch Grenzen und Barrieren vorhanden sind.

Es ist oft mehr Orientierung, Strukturierung und eine stärkere Stringenz notwendig. Unter Umständen müssen erst (Lern-)Strategien erarbeitet werden, bevor eine eher offene Form der selbstständigen Auseinandersetzung mit der Thematik stattfinden kann. Die Möglichkeiten der Unterstützung sind vielfältig, aber sie müssen bekannt sein, auf die Bedarfe der Schüler/-innen angepasst sein und auch genutzt werden. Vielleicht muss das Lernsetting etwas stärker geschlossen werden und zumindest teilweise zuerst mit anderen Methoden als einer offenen Projektarbeit gestartet werden.

Zudem ist eine Differenzierung der Ziele möglich und sogar in einigen Fällen notwendig: mehrere kleine und gut zu erreichende Ziele (möglichst auch gut visualisiert zur Orientierung), statt ein großes und zu weit entferntes Ziel. An die klar formulierten und erreichbaren Ziele sollte ein aufmunterndes und motivierendes Feedback gebunden sein (differenzierte Perspektiven zu Feedback in der inklusiven Bildung vgl. Vierbuchen \& Bartels, 2019). Die einzelnen Schritte, die vollzogen werden müssen, um sich etwas zu erarbeiten oder ein Ziel zu erreichen, müssen oft noch deutlicher formuliert werden. Auch bei BNE ist natürlich zieldifferenter Unterricht möglich und teilweise notwendig. Bei heterogenen Lerngruppen ist es nicht immer möglich, dass alle das gleiche Ziel erreichen. Allerdings sollte diese Differenzierung kein Selbstzweck sein und nicht zu einem Selektionsinstrument werden (Böhme, 2019). Denkbar sind auch die gemeinsame Erarbeitung und Verschriftlichung einer Checkliste, die einzelne Schüler/-innen in den weiteren Prozess der Auseinandersetzung mitnehmen. Für den Einstieg kann auch eine Schritt-für-Schritt-Anleitung, die Schülerinnen und Schüler abarbeiten können, sehr hilfreich sein.

$\mathrm{Zu}$ diskutieren wäre ebenfalls eine stärkere Produktund Erfahrungsorientierung zur Erarbeitung oder auch Ergebnissicherung, statt rein kognitive, gesprächsorientierte Bearbeitung von Aspekten. Weiterhin können vor allem für Lernende mit kognitiven Beeinträchtigungen affektiv orientierte Zugän- 
ge zu Fragen einer nachhaltigen Entwicklung ermöglicht werden: Dies kann z.B. durch Musik, kreative Zugänge wie Zeichnen, Naturbeobachtung mit allen Sinnen oder über Gefühle erfolgen (Böhme, 2019). Immer wiederkehrende Feedbackschleifen während des Prozesses unterstützen manche Schüler/-innen zusätzlich. Dies sind nur einige mögliche Ansatzpunkte, die jedoch bereits ein breites Spektrum an Hilfestellung und Ansätze zur Differenzierung bieten.

Trotz aller Chancen und Möglichkeiten der Differenzierung stellt der Umgang mit Komplexität und Unsicherheit eine besondere Herausforderung dar (Böhme, 2019). Denn BNE zeichnet sich gerade durch die Arbeit mit hochkomplexen Themen (z.B. Globalisierung, Klimawandel) aus. Um allen Lernenden einen Zugang zu diesen Themen zu ermöglichen, sind besondere Strategien der Komplexitätsreduktion erforderlich. Dazu gehören u.a. ein ausgeprägter Lebensweltbezug sowie die Verwendung leichter Sprache (Böhme, 2019; Führing \& Böhme, 2015), so „dass eine tatsächliche Durchdringung des Inhalts von allen SchülerInnen geleistet werden kann" (Führing \& Böhme, 2015, S. 17).

Die obigen Ausführungen machen deutlich: Um eine BNE inklusiv gestalten zu können, muss zunächst und immer wieder während des Prozesses die konkrete Zielgruppe analysiert werden. Wie heterogen ist die Gruppe, in Bezug auf welche Aspekte? Welche Bedarfe an (sonderpädagogischer) Unterstützung bestehen? Welche Sprachen werden gesprochen? Welche Strukturierungshilfen sind gefordert? Wie müssen das Lernsetting und die Materialien aufbereitet und angelegt sein, damit alle Schüler/-innen die Möglichkeit haben, das Ziel zu erreichen und am Prozess zu partizipieren? Oder muss das Ziel bei einigen angepasst werden, gerade um die Partizipation zu stärken? Eine solche differenzierte Bearbeitung von Themen in heterogenen Gruppen kann dabei als Bereicherung für den Bildungsprozess betrachtet werden (Böhme, 2019). Denn die Anpassung des Vorgehens und des Materials spielt nicht nur für Schüler/-innen mit besonderen Bedarfen eine Rolle, sondern es profitieren alle Beteiligten, wenn inklusive Bildung adäquat umgesetzt wird (European Agency for Development in Special Needs Education, 2014). „Einigen SchülerInnen wird das vernetzte Denken schwerfallen, so dass sie Differenzierungsmaßnahmen und emotionale, empathische Zugänge benötigen, die aber auch für andere SchülerInnen eine Erweiterung und Vertiefung ihres Erfahrungshorizontes bedeuten“ (Führing \& Böhme, 2015, S. 18). Es geht mithin um einen positiven und produktiven Umgang mit Vielfalt (Böhme, 2019; Böhme \& Führing, 2014), der jedoch nicht intuitiv von den Lehrkräften umgesetzt werden kann, sondern einiges an Know-how benötigt.

Für die Gestaltung einer inklusiven BNE können folgende Prinzipien benannt werden (nach bezev, 2019; Böhme, 2019; Engagement Global \& bezev, 2017; Führing \& Böhme, 2015; Westermeier, 2015):

- Anknüpfen an ein aktuelles Thema, das den Schülerinnen und Schülern bekannt ist und das für sie Relevanz im Alltag besitzt (Lebensweltbezug)

- Möglichkeiten zum praktischen Arbeiten, z.B. konkretes Material und möglichst wenig rein kognitiv-abstrakte Aspekte (Handlungsorientierung)
- gemeinsames Arbeiten in heterogenen Lerngruppen; evtl. durch den Einsatz peergestützter Verfahren

- differenzierte Aufgaben je nach Fähigkeiten oder Förderbedarf (Binnendifferenzierung) und Komplexitätsreduktion

- kognitive und affektiv-orientierte Kompetenzförderung, z.B. durch vorbereitende Phasen des Übens von Strategien, aber auch begleitende Hilfestellungen und Rückmeldungen

- multisensorisches Arbeiten: verschiedene Sinne ansprechen, ohne jedoch zu überfordern. Unter Umständen eher wiederholend nacheinander verschiedene Zugänge ermöglichen als parallel

- Perspektivenwechsel: sich über die eigenen Werte und die Werte anderer Menschen bewusst werden, sich in andere Menschen hineinzuversetzen und ihre Werte zu würdigen

- Förderung der Fähigkeit zur Empathie mit anderen Lernenden sowie mit Menschen in anderen Ländern und der Natur

Im Sinne des Whole School Approach sollten BNE und Inklusion jedoch nicht nur Eingang in den Unterricht finden, sondern Grundlage für eine umfassende Veränderung der Institution Schule sein (bezev, 2019; Böhme, 2019). Dies kann sich u.a. auf die nachhaltige und barrierefreie Gestaltung des Schulgeländes und -gebäudes sowie ein nachhaltiges und vielfältiges Angebot in der Schulmensa oder dem Schulkiosk, das von allen gemeinsam gestaltet und allen gerecht wird, beziehen. Dies ermöglicht allen Schüler/-innen einen vielfältigen und ganzheitlichen Zugang zu Fragen der Nachhaltigkeit und Inklusion. Ihnen werden praktische Erfahrungsmöglichkeiten geboten. Außerdem können die Schüler/-innen selbst an der Gestaltung der Schule beteiligt werden, Verantwortung übernehmen und dabei ihre (heterogenen) Perspektiven einbringen. Nicht zuletzt übernimmt die Schule mit der umfassenden Berücksichtigung von Nachhaltigkeits- und Inklusionskriterien eine Vorbildfunktion. Für die Umsetzung eines Whole School Approach sind die Unterstützung der Schulleitung sowie die Einbindung des gesamten Kollegiums grundlegend; zudem können die Einrichtung einer Steuerungsgruppe und die Zusammenarbeit mit Nichtregierungsorganisationen oder Partner/-innen aus Ländern des Globalen Südens förderlich sein (Böhme, 2019; Führing \& Böhme, 2015). Hier sollte sich das vorhandene schulische Netzwerk erweitern und die Kooperation gestärkt werden.

Sowohl BNE als auch inklusive Bildung stellen hohe Anforderungen an die Lehrenden und deren Professionalisierung (European Agency for Development in Special Needs Education, 2011, 2014; Bertschy et al., 2013; Vare et al., 2019). Im Rahmen der Lehrerbildung müssen die Lehrkräfte sowohl Wissen über die Konzepte BNE und inklusive Bildung als auch Kompetenzen zu deren Umsetzung erwerben. Zur Umsetzung der oben genannten Möglichkeiten zur Differenzierung und Modifikation der Methoden oder Materialien ist ein breites Spektrum an Wissen und Können notwendig. Zudem gilt für beide Konzepte, dass eine positive Einstellung gegenüber einer nachhaltigen Entwicklung bzw. Inklusion einer Arbeit mit den pädagogischen Konzepten förderlich ist. Hier besteht für alle drei Phasen der Lehrerbildung Potenzial für eine Verbindung beider Konzepte. 


\section{Schlussfolgerungen und Ausblick}

Zusammenfassend ist festzustellen, dass sowohl BNE als auch Inklusion ein hohes Potenzial besitzen, gemeinsam gedacht und umgesetzt zu werden. Dabei ist Inklusion als Voraussetzung für BNE zu bewerten. Eine inklusive BNE ist hochkomplex und muss gut geplant und umgesetzt werden. Die Anforderungen an Lehrende sind hoch. Jedoch können beide Konzepte sehr gut in Zusammenhang gebracht werden und die Verbindung von BNE und Inklusion scheint für beide Konzepte absolut gewinnbringend zu sein. Es ist ein gemeinsamer normativer Kern vorhanden. Die inklusive methodisch-didaktische Gestaltung stellt für alle Lernenden einen Gewinn dar. Eine inklusive BNE als Whole Institution Approach in der Schule erscheint vielversprechend für eine positive Gestaltung des Schulalltags und verspricht eine positive Wirkung in die Gesellschaft. Denn sowohl Lehrkräfte als auch Schüler/-innen können so gegenseitig und in ihren sozialen Kontext über den schulischen Bereich hinaus als positive Modelle dienen. Für die (Weiter-)Entwicklung einer inklusiven BNE wird es erforderlich sein, mehr Materialien für die Bildungspraxis zu entwickeln sowie in diesem Beitrag skizzierte Ansätze - unter wissenschaftlicher Begleitung - in der Praxis zu erproben.

\section{Anmerkungen}

1 Siehe: https://www.bezev.de/de/home/bildung-fuer-nachhaltige-entwicklung/bnein-der-schule/material/

\section{Literatur}

Baumert, B., Vierbuchen, M.-C. \& Team BRIDGES (2018). Eine Schule für alle - Wie geht das? Qualitätsmerkmale und Gelingensbedingungen für eine inklusive Schule und inklusiven Unterricht. Zeitschrift für Heilpädagogik, 69(11), 526-541. Zugriff am 24.01.2020 https://www.uni-vechta.de/fileadmin/user_upload/Zent rum_fuer_Lehrerbildung/ProjektBridges/02_Dateien/zfh_11.2018_baumert-vierbuchen.pdf

Bertschy, F., Künzli, C. \& Lehmann, M. (2013). Teachers' Competencies for the Implementation of Educational Offers in the Field of Education for Sustainable Development. Sustainability, 5(12), 5067-5080. https://doi.org/10.3390/su5125 067

bezev - Behinderung und Entwicklungszusammenarbeit e.V. (2019). Bildung für nachhaltige Entwicklung inklusiv als Aufgabe der ganzen Schule. Eine Handreichung mit praktischen Anregungen für Grundschulen. Essen: bezev.

Bhatia, S. \& Singh, S. (2015). Creating a Sustainable and Inclusive Future through Youth Action and Participation. Behinderung und internationale Entwicklung, 26(2), Inklusion in der Bildung für nachhaltige Entwicklung, 29-34. Zugriff am 24.01.2020 http://www.zbdw.de/projekt01/media/pdf/2015_2_BIE.pdf

Böhme, L. (2018). Keine Transformation ohne Teilhabe! Globales Lernen im Kontext gesellschaftlicher Inklusion. In VENRO (Hrsg.), Globales Lernen: Wie transformativ ist es? Impulse, Reflexionen, Beispiele. Diskussionspapier 2018 (S. 51-53). Berlin: VENRO. Zugriff am 24.01.2020 https://venro.org/fileadmin/user_upload/ Dateien/Daten/Publikationen/Diskussionspapiere/2018_Globales_Lernen.pdf

Böhme, L. (2019). Politische Bildung für Schülerinnen und Schüler mit sonderpädagogischem Förderbedarf. Perspektiven Globalen Lernens an Förderzentren. Frankfurt am Main: Wochenschau.

Böhme, L. \& Führing, G. (2014). Globales Lernen als Katalysator für Diversity und Inklusion. In Verein niedersächsischer Bildungsinitiativen e.V. (Hrsg.), Die große Globalisierung für kleine Leute - Globales Lernen mit Grundschulkindern (S. XIIIXV). Barnstorf: VNB. Zugriff am 24.01.2020 https://www.bizme.de/documents/ VNB_Broschuere_Webansicht_NRO.pdf

Engagement Global \& bezev (2017). Cashew - a global learning challenge. Learning material for inclusive education. Bonn. Zugriff am 24.01.2020 http://www.esd-expert.net/files/ESD-Expert/pdf/Was_wir_tun/Lehr-\%20und\%20Lernmaterialien/ Cashew_South\%20Africa.pdf

European Agency for Development in Special Needs Education (2011). Inklusionsorientierte Lehrerbildung in Europa. Chancen und Herausforderungen. Zugriff am 24.01.2020 https://www.european-agency.org/sites/default/files/te4i-synthesis-rep ort-de.pdf
European Agency for Development in Special Needs Education (2014). Fünf Kernaussagen in Bezug auf inklusive Bildung. Von der Theorie zur Praxis. Zugriff am 24.01.2020 https://www.european-agency.org/sites/default/files/Five_Key_Messa ges_for_Inclusive_Education_DE.pdf

Führing, G. \& Böhme, L. (2015). Globales Lernen inklusiv?! Theoretische und praxisrelevante Überlegungen. Berlin: BGZ Berliner Gesellschaft für internationale Zusammenarbeit mbH. Zugriff am 24.01.2020 https://www.bgz-berlin.de/files/broschuere_globales_lernen_inklusiv_global_fairness_1-min.pdf

Gable, R. A., Tonelson, S. W., Park, K. L., Sheth, M., \& Wilson, C. (2012). Importance, Usage, and Preparedness to Implement Evidence-based Practices for Students with Emotional Disabilities: A Comparison of Knowledge and Skills of Special Education and General Education Teachers. Education and Treatment of Children, 35(4), 499-519. https://doi.org/10.1353/etc.2012.0030

Hennemann, T., \& Hillenbrand, C. (2010). Klassenführung - Classroom Management. In B. Hartke, K. Koch \& K. Diehl (Hrsg.), Förderung in der schulischen Eingangsstufe (S. 255-279). Stuttgart: Kohlhammer.

KMK - Kultusministerkonferenz \& BMZ - Bundesministerium für wirtschaftliche Zusammenarbeit und Entwicklung (2015). Orientierungsrahmen für den Lernbereich Globale Entwicklung im Rahmen einer Bildung für nachhaltige Entwicklung. Berlin \& Bonn. Zugriff am 24.01.2020 http://www.kmk.org/fileadmin/veroeffentlichungen _beschluesse/2015/2015_06_00-Orientierungsrahmen-Globale-Entwicklung.pdf Künzli David, C. (2007). Zukunft mitgestalten. Bildung für eine nachhaltige Entwicklung - Didaktisches Konzept und Umsetzung in der Grundschule. Bern: Haupt.

Littledyke, M. \& Manolas, E. (2011). Education for Sustainability Pedagogy: Ideological and Epistemological Barriers and Drivers. In W. Leal Filho (Hrsg.), World trends in education for sustainable development (S. 77-104). Frankfurt a.M.: Springer. Michelsen, G. \& Fischer, D. (2015). Bildung für nachhaltige Entwicklung. Wiesbaden: Hessische Landeszentrale für politische Bildung. https://doi.org/10.2307/j. ctvd7w8kk.12

Rieckmann, M. (2016). Bildung für nachhaltige Entwicklung - Konzeptionelle Grundlagen und Stand der Implementierung. In M. Schweer (Hrsg.), Bildung für nachhaltige Entwicklung in pädagogischen Handlungsfeldern - Grundlagen, Verankerung und Methodik in ausgewählten Lehr-Lern-Kontexten (S. 11-32). Frankfurt a.M.: Peter Lang.

Rieckmann, M. (2018). Chapter 2 - Learning to transform the world: key competencies in ESD. In A. Leicht, J. Heiss \& W. J. Byun (Hrsg.), Issues and trends in Education for Sustainable Development (S. 39-59). Paris: UNESCO. Zugriff am 24.01.2020 http://unesdoc.unesco.org/images/0026/002614/261445E.pdf

Rieckmann, M., Fischer, D. \& Richter, S. (2014). Nachhaltige Ernährung im Wertediskurs - Beiträge einer Hochschulbildung für nachhaltige Entwicklung. In C. Schank, K. Vorbohle \& J. H. Quandt (Hrsg.), Perspektive Nahrungsmittelethik (S. 29-58). München \& Mering: Rainer Hampp.

Rieckmann, M. \& Schank, C. (2016). Sozioökonomisch fundierte Bildung für nachhaltige Entwicklung - Kompetenzentwicklung und Werteorientierungen zwischen individueller Verantwortung und struktureller Transformation. SOCIENCE, 1(1), 65-79.

Rieckmann, M. \& Stoltenberg, U. (2011). Partizipation als zentrales Element von Bildung für eine nachhaltige Entwicklung. In K. Kuhn, J. Newig \& H. Heinrichs (Hrsg.), Nachhaltige Entwicklung? Welche Rolle für Partizipation und Kooperation? (S. 119-131). Wiesbaden: VS Verlag. https://doi.org/10.1007/978-3-531-93020$6 \_8$

Svinos, M. (2019). Inklusive Bildung. Gemeinsam für eine nachhaltige Entwicklung. ökopadNEWS, ANU-Informationsdienst Umweltbildung, Nr. 301, 29. Zugriff am 24.01.2020 https://www.umweltbildung.de/8321.html? \& fontsize=2

UNESCO - United Nations Educational, Scientific and Cultural Organization (2017). Education for Sustainable Development Goals. Learning Objectives. Paris: UNESCO.Zugriffam 24.01.2020 http://unesdoc.unesco.org/images/0024/002474 1247 444e.pdf

United Nations (2006). UN-Convention on the Rights of Persons with Disabilities. Zugriff am 24.01.2020 http://www.un.org/disabilities/convention/conventionfull. shtm

Vare, P., Arro, G., de Hamer, A., Del Gobbo, G., de Vries, G., Farioli, F. et al. (2019). Devising a Competence-Based Training Program for Educators of Sustainable Development: Lessons Learned. Sustainability, 11(7). https://doi.org/10.3390/su1107 1890

Vare, P. \& Scott, W. (2007). Learning for a Change: Exploring the Relationship between Education and Sustainable Development. Journal of Education for Sustainable Development, 1(2), 191-198.https://doi.org/10.1177/097340820700100209 Vierbuchen, M.-C. \& Bartels, F. (Hrsg.) (2019). Erfolgreiches Feedback - Wie kann Feedback in Schule und Unterricht unterstützend wirken? Stuttgart: Kohlhammer.

Wals, A. E. J. \& Lenglet, F. (2016). Sustainability citizens: Collaborative and disruptive social learning. In R. Horne, J. Fien, B. B. Beza \& A. Nelson (Hrsg.), Sustainability Citizenship in Cities: Theory and Practice (S. 52-66). London: Routledge. 
WBGU - Wissenschaftlicher Beirat der Bundesregierung Globale Umweltveränderungen (2011). Welt im Wandel: Gesellschaftsvertrag für eine große Transformation. Berlin: WBGU.

Weinert, F. E. (2001). Leistungsmessungen in Schulen. Weinheim: Beltz.

Westermeier, C. (2015): Inklusives und Globales Lernen mit dem Material von bezev zum Thema Klima und zum Thema Wasser. Behinderung und internationale Entwicklung, 26(2), Inklusion in der Bildung für nachhaltige Entwicklung, 22-28. Zugriff am 24.01.2020 http://www.zbdw.de/projekt01/media/pdf/2015_2_BIE. pdf

\section{Dr. ${ }^{\text {in }}$ Marie-Christine Vierbuchen}

ist Juniorprofessorin für Inklusive Bildung im Fach Erziehungswissenschaften der Fakultät I - Bildungs- und Gesellschaftswissenschaften an der Universität Vechta. Sie ist die wissenschaftliche Leitung des Graduiertenzentrums der Universität Vechta und Mitglied im Vorstand des Zentrums für Lehrerbildung an der Universität Vechta. Sie arbeitet in mehreren Vorständen in der Praxis (Verband Sonderpädagogik - vds, Lebenshilfe Delmenhorst und Landkreis Oldenburg) und begleitet Schulen auf dem Weg zur Inklusion. Ihre Arbeitsschwerpunkte sind Lehrkräfteprofessionalisierung für inklusive Bildung, Diagnostik und Förderung bei Lern- und Verhaltensbeeinträchtigungen, Classroom Management, sozial-kognitive Informationsverarbeitung und Schulabsentismus und Dropout.

\section{Dr. Marco Rieckmann}

ist Professor für Hochschuldidaktik, Schwerpunkt Schlüsselkompetenzen, im Fach Erziehungswissenschaften der Fakultät I - Bildungs- und Gesellschaftswissenschaften an der Universität Vechta. Er ist Nachhaltigkeitsbeauftragter der Universität Vechta, Vertreter der Deutschen Gesellschaft für Erziehungswissenschaft (DGfE) im Council der European Educationa Research Association (EERA) sowie Sprecher des Deutschsprachigen Netzwerks „LehrerInnenbildung für nachhaltige Entwicklung“ (LeNa). Seine Arbeitsschwerpunkte sind: Hochschuldidaktik, (Hochschul-)Bildung für nachhaltige Entwicklung, Nachhaltige Hochschulentwicklung.

\section{UNSERE BUCHEMPFEHLUNG}

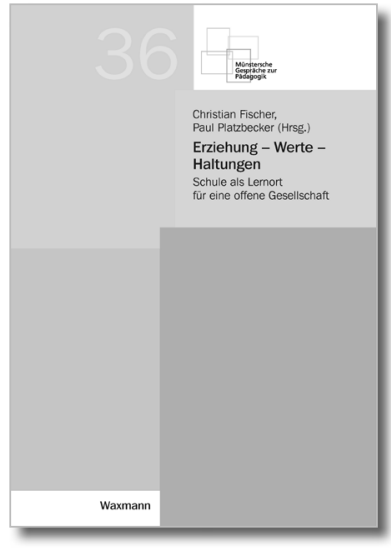

Münstersche Gespräche zur Pädagogik, Band 36 2020, 170 Seiten, br., 17,90€, ISBN 978-3-8309-4143-9

E-Book: 16,99€, ISBN 978-3-8309-9143-4

\section{Christian Fischer, Paul Platzbecker (Hrsg.) \\ Erziehung - Werte - Haltungen \\ Schule als Lernort für eine offene Gesellschaft}

Wirtschaftliche und soziale Auswirkungen von Globalisierung und Digitalisierung erzeugen komplexe Problemlagen. Einfache Antworten von Populisten und Autokraten erscheinen in dieser Situation auch jungen Menschen mitunter attraktiver als langwierige gesellschaftliche Diskurse.

Einerseits kommen demokratische Gesellschaften nicht ohne eine geteilte Wertebasis aus. Andererseits besteht ihr Ethos gerade darin, dass sie der Offenheit und Toleranz verpflichtet sind. Demokratie ist daher eine Lebensform, die man lernen muss.

Kann sich Schule als Lernort für eine offene Gesellschaft erweisen? Dieser Themenband lotet Möglichkeiten und Grenzen einer Erziehung zu demokratischem Handeln aus. Neben wissenschaftlichen Referaten werden aktuelle Ansätze aus der Praxis vorgestellt und diskutiert. 